\title{
Mechanical analysis of symmetrical driving the movement of the lifting platform
}

\author{
Sun $\mathrm{Yi}^{1, \text { a }}$, Liu Yuancai ${ }^{2, \mathrm{~b}}$ and Xia Binghua ${ }^{3, \mathrm{c}}$ \\ ${ }^{1}$ Experimentalist, Experimental Teaching Center of Provinical Structural Engineering of Civil and \\ Engineering, Southwest forestry university, China \\ ${ }^{2}$ Professor ,Dept. of Civil and Engineering, Southwest forestry university, China \\ ${ }^{3}$ Experimentalist, Experimental Teaching Center of Provinical Structural Engineering of Civil and \\ Engineering, Southwest forestry university, China \\ asunyi3@126.com, byuancailiu@126.com, cmzatamd@126.com
}

Keywords: Fork lifting platform, Hinge axis shear force, Motion analysis.

\begin{abstract}
This paper is to mainly study to the lifting platform in the unit by the board device in the copper electrolysis anode plate. According to the design and application requirements, It is proposed four-cylinder symmetrical drive overloaded scissor lift platform design and mechanical analysis of the lifting platform which come to the cylinder driving force simply hinged single cylinder drive scissor lift platform 1/4. If the four cylinder mounting included angles with the horizontal line is equal, intermediate hinge shaft shear stress calculation for zero,it is very helpful for heavy load condition, and the main components for the parameters determination and strength check.
\end{abstract}

\section{Introduction}

Scissors hydraulic lifting platform is a shearing fork type mechanical structure, the lifting platform lifting has high stability, big bearing capacity, wide working platform ${ }^{[1,2]}$. With compact structure, small installation space, good maneuverability and high degree of automation and is easy to realize the centralized control and other features, and is suitable for people to work at the same time, make the ground operations on a larger scale, more effective, safer, is widely used in every field of the society.

\section{Use and design requirements}

This paper studies the lifting platform is the metallurgical equipment in the application of the anode plate preparation unit by the plate portion of the lifting platform, as shown in Figure 1, copper electrolytic anode plate preparation unit is divided into the overall by the portion of the plate, transverse moving section, row plate part $^{[1]}$.

Work process: (1) starting unit, the lift platform to rise to 2 after receiving highly, bolt component 3 will be launched after the pin, preventing forklift plate plate forward dumping. (2)forklift anode plate raised and placed on the lifting platform 2, forklift whereabouts and exit;(3) on cylinder in 4 from both sides launch, make plate edge alignment, center line in 3, after the latch assembly bolt back; the lifting platform 2 decline to make plate hanging to the conveyor chain in 5 , the board process completed.

According to the requirement, the shaping of the copper anode plate $25 \pm 2$ block by forklift truck into the unit by the board on the station. Raw material specifications: size is $870 \times 840 \mathrm{~mm}$; anode plate thickness is $42 \pm 3 \mathrm{~mm}$; anode plate weight is $260 \pm 5 \mathrm{~kg}$ / block. By receiving and conveying chain is highly restricted, this paper studies the lifting platform must satisfy is the main parameter:

The minimum distance from the ground: $700 \mathrm{~mm}$;

Material receiving height: $950 \mathrm{~mm}$;

Maximum lifting height: $250 \mathrm{~mm}$;

Table size ( length $\times$ width ) $: 1450 \mathrm{~mm} \times 1100 \mathrm{~mm}$;

The minimum distance from the ground when the lifting force ( $10 \mathrm{Mpa}): 8 \mathrm{t}$. 
From the design parameters, the lifting platform has a large quantity of load capacity, small occupied space, convenient operation, the lifting speed characteristics.

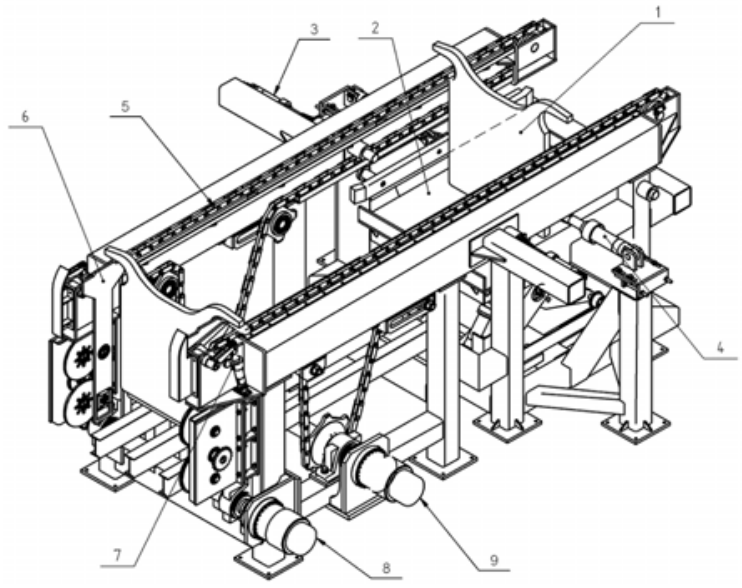

1-anode plate; 2-lifting platform; 3-After the bolt assembly; 4- right in the components; 5-The conveyor chain; 6-Plate lifting mechanism; 7-Fragmentation mechanism; 8-Lift oil motor; 9-The conveyor chain drive motor

Fig 1 The portion of the plate structure

\section{Four cylinder symmetrical driving heavy fork lifting platform motion analysis}

Scissors elevating platform main body structure is in the form of scissors, rely on the fork arm of the unfolded and folded to realize cargo lifting function, which is powered by the extension of the oil cylinder to drive the scissors elevating platform unfolding and folding, lifting has higher stability, mechanical shearing fork type structure makes the lifting platform is wide and large bearing capacity. Four cylinder symmetrical driving heavy fork lifting platform plane diagram as shown in Figure 2 and Figure 3 , by two cylinder drive and is symmetrical, to realize the lifting platform, namely four cylinder. The cylinder is symmetrically distributed, in driving process, requiring four cylinder synchronous motor. $\mathrm{S}$ is a cylinder end to fork arm distance, $\mathrm{T}$ is inside the fork arm end distance, $\mathrm{E}$ is the cylinder push rod end to the lower end of the fork arm by distance, structure analysis:

$S^{2}=T^{2}+E^{2}-2 T E \cos (\theta+\alpha)$

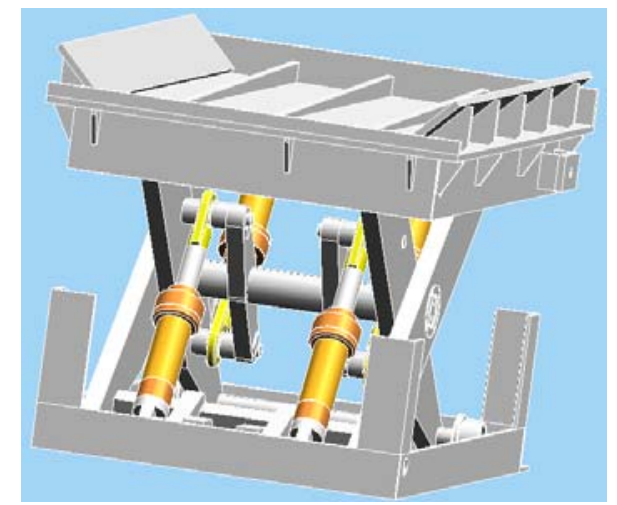

Fig.2 Heavy duty driving four cylinder scissors elevating platform We can obtain:

$$
\cos (\theta+\alpha)=\frac{T^{2}+E^{2}-S^{2}}{2 T E}
$$

When the cylinder push rod change $\Delta \mathrm{S}$, there are:

$(S+\Delta S)^{2}=T^{2}+E^{2}-2 T E \cos (\theta+\Delta \theta+\alpha)$

We can obtain:

(1)

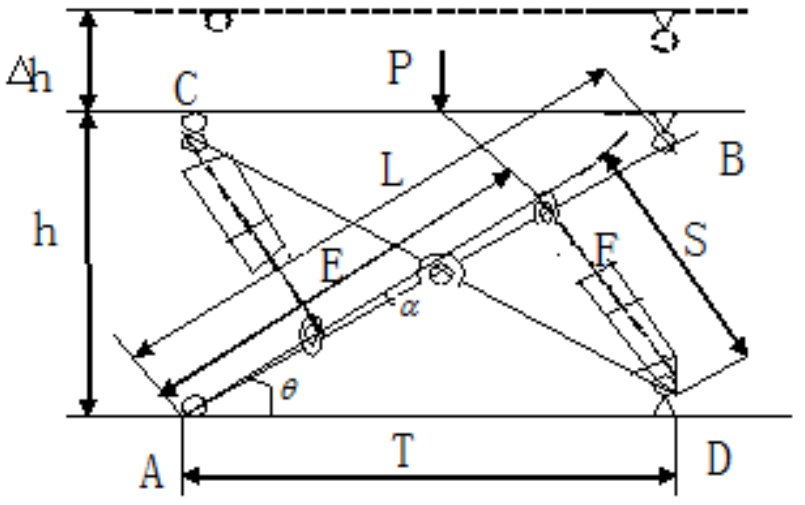

Fig 3 Four cylinder symmetrical driving heavy fork lifting platform. 
$\cos (\theta+\Delta \theta+\alpha)=\frac{T^{2}+E^{2}-(S+\Delta S)^{2}}{2 T E}$

By Eq .2 subtracted Eq.3, We can obtain:

$$
\begin{aligned}
& \cos (\theta+\Delta \theta+\alpha)-\cos (\theta+\alpha)=-2 \sin \left(\theta+\alpha+\frac{\Delta \theta}{2}\right) \sin \left(\frac{\Delta \theta}{2}\right) \\
& =\frac{T^{2}+E^{2}-(S+\Delta S)^{2}}{2 T E}-\frac{T^{2}+E^{2}-S^{2}}{2 T E}=\frac{S^{2}-(S+\Delta S)^{2}}{2 T E}
\end{aligned}
$$

We can obtain:

$$
\begin{aligned}
& \frac{(S+\Delta S)^{2}-S^{2}}{2 T E}=2 \sin \left(\theta+\alpha+\frac{\Delta \theta}{2}\right) \sin \left(\frac{\Delta \theta}{2}\right) \\
& \sin \left(\frac{\Delta \theta}{2}\right)=\frac{(S+\Delta S)^{2}-S^{2}}{2 T E\left[2 \sin \left(\theta+\alpha+\frac{\Delta \theta}{2}\right)\right]}
\end{aligned}
$$

From the principle of virtual work ${ }^{[4-6]}$,We can obtain:

$P * \Delta h=\left(F_{1}+F_{2}+F_{3}+F_{4}\right) * \Delta S$

$\mathrm{F} 1, \mathrm{~F} 2, \mathrm{~F} 3, \mathrm{~F} 4$ are four cylinder thrust; the lifting platform lifting height of the $\Delta \mathrm{h}$ is:

$\Delta h=L \sin (\theta+\Delta \theta)-L \sin \theta=2 L \cos \left(\theta+\frac{\Delta \theta}{2}\right) \sin \left(\frac{\Delta \theta}{2}\right)$

Substituting Eq.5, We can obtain:

$$
P * 2 L \cos \left(\theta+\frac{\Delta \theta}{2}\right) \sin \left(\frac{\Delta \theta}{2}\right)=\left(F_{1}+F_{2}+F_{3}+F_{4}\right) * \Delta S
$$

Because the four cylinder synchronous movement, in order to ensure the smooth upgrade platform, four cylinder push rod retractable equal distance, according to the Eq.4 derivation, knowable:

$$
\sin \left(\frac{\Delta \theta}{2}\right)=\frac{(S+\Delta S)^{2}-S^{2}}{2 T E\left[2 \sin \left(\theta+\alpha+\frac{\Delta \theta}{2}\right)\right]}
$$

Substitution Eq.6, We can obtain:

$$
\begin{aligned}
& \left(F_{1}+F_{2}+F_{3}+F_{4}\right) * \Delta S=\frac{P * L \cos \left(\theta+\frac{\Delta \theta}{2}\right) *\left(2 S \Delta S+\Delta S^{2}\right)}{2 T E \sin \left(\theta+\alpha+\frac{\Delta \theta}{2}\right)} \\
& \left(F_{1}+F_{2}+F_{3}+F_{4}\right)=\frac{2 P * L * S \cos \left(\theta+\frac{\Delta \theta}{2}\right) * \Delta S}{2 T E \sin \left(\theta+\alpha+\frac{\Delta \theta}{2}\right) \Delta S}+\frac{P * L \cos \left(\theta+\frac{\Delta \theta}{2}\right) * \Delta S^{2}}{2 T E \sin \left(\theta+\alpha+\frac{\Delta \theta}{2}\right) \Delta S} \\
& \text { Order: } \quad G_{1}=\frac{2 P * L * S \cos \left(\theta+\frac{\Delta \theta}{2}\right) * \Delta S}{2 T E \sin \left(\theta+\alpha+\frac{\Delta \theta}{2}\right) \Delta S}, G_{2}=\frac{P * L \cos \left(\theta+\frac{\Delta \theta}{2}\right) * \Delta S^{2}}{2 T E \sin \left(\theta+\alpha+\frac{\Delta \theta}{2}\right) \Delta S}
\end{aligned}
$$

$$
\frac{G 2}{G 1}=\frac{\frac{P * L \cos \left(\theta+\frac{\Delta \theta}{2}\right) * \Delta S^{2}}{2 T E \sin \left(\theta+\alpha+\frac{\Delta \theta}{2}\right) \Delta S}}{\frac{2 P * L * S \cos \left(\theta+\frac{\Delta \theta}{2}\right) * \Delta S}{2 T E \sin \left(\theta+\alpha+\frac{\Delta \theta}{2}\right) \Delta S}}=\frac{\Delta S}{2}
$$

When $\lim \Delta \theta \rightarrow 0, \mathrm{G}_{2} / \mathrm{G}_{1}=\Delta \mathrm{S} / 2 \rightarrow 0, \mathrm{G} 2$ is $\mathrm{G} 1$ the infinitesimal of higher order ${ }^{[7]}$, therefore: 


$$
\left(F_{1}+F_{2}+F_{3}+F_{4}\right)=\frac{2 P * L * S \cos \left(\theta+\frac{\Delta \theta}{2}\right) * \Delta S}{2 T E \sin \left(\theta+\alpha+\frac{\Delta \theta}{2}\right) \Delta S}=\frac{2 P * L * S \cos \left(\theta+\frac{\Delta \theta}{2}\right)}{2 T E \sin \left(\theta+\alpha+\frac{\Delta \theta}{2}\right)}
$$

When $\lim \Delta \theta \rightarrow 0$ :

$$
\left(F_{1}+F_{2}+F_{3}+F_{4}\right)=\frac{P * L * S * \cos \theta}{T E \sin (\theta+\alpha)}
$$

Fork lifting platform is symmetrically distributed mechanism, to ensure the four cylinder synchronous movement, in the design, the four type hydraulic cylinders are symmetrically distributed in the lifting process, then $\mathrm{F} 1=\mathrm{F} 2=\mathrm{F} 3=\mathrm{F} 4$.

Substitution Eq.7,We can obtain:

$$
\begin{aligned}
& 4 F=\frac{P * L * S * \cos \theta}{T E \sin (\theta+\alpha)} \\
& F=\frac{P}{4} * \frac{L * S * \cos \theta}{T E \sin (\theta+\alpha)}
\end{aligned}
$$

Eq.8, F refers to a single thrust of cylinder.

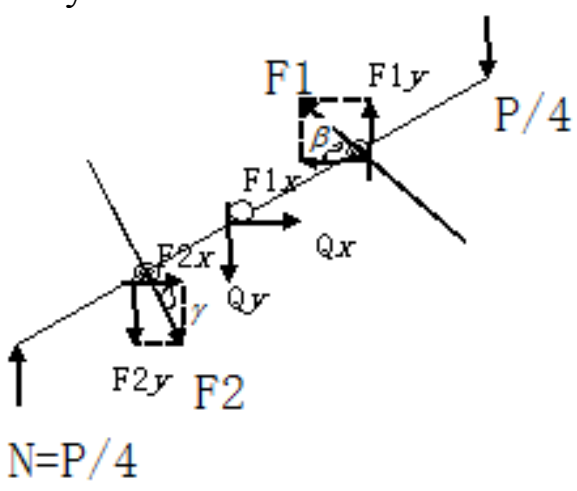

Fig 4 Swing rod fork arm AB force analysis diagram

The inner fork arm by four cylinder for pushing, complex stress, below I on the swing rod fork arm $\mathrm{AB}$ stress analysis, as shown in Figure4, can be obtained by force analysis of hinge axis shear size relationship:

$$
\begin{aligned}
& \sum F_{X}=0 ; \quad \sum F_{Y}=0 \\
& F_{1 x}=F_{1} \cos \beta \\
& F_{2 x}=F_{2} \cos \gamma
\end{aligned}
$$

We can obtain:

$$
F_{1 x}=F_{2 x}+Q_{x}
$$

Substitution Eq.9 and Eq.10 into Eq.11,We can obtain:

$$
F_{1} \cos \beta=F_{2} \cos \gamma+Q_{x}
$$

It is known $\mathrm{F} 1=\mathrm{F} 2$; the hinge shaft shear force $Q=Q_{x}=F_{1} \cos \beta-F_{2} \cos \gamma$; when the cylinder push rod and horizontal angles equal $(\beta=\gamma)$, We can obtain $\mathrm{Q}=0$.

Similarly obtain:

$Q_{Y}=0$

It is easy to achieve,to ensure that the four cylinder and the horizontal angle is equal in the installation of production process.

\section{Conclusions}

It is knowable Through Eq. 8 four cylinder symmetrical driving heavy fork lifting platform cylinder thrust just single cylinder lifting platform $1 / 4$ bearing the same load, especially when the load is heavy load conditions, articulated single cylinder driven shearing fork lifting platform must 
manufacture of large diameter cylinder, increasing costs, occupancy large space. Four cylinder symmetrical driving heavy fork lifting platform can be used for hydraulic synchronous motion method to improve the hydraulic payload, is a feasible method.

From the Eq.12 derivation shows, If the four hydraulic cylinder and a horizontal hinge axis equal included angle, shear stress calculation is zero, will greatly enhance the performance and service life of the hinge shaft. So the four cylinder symmetrical driving heavy fork lifting platform than the commonly used horizontally driven scissors lift platform, articulated single cylinder driven shearing fork lifting platform has many advantages, for heavy haul fork lifting platform provide a wider development train of thought.

\section{Acknowledgements}

This work was financially supported by the Provincial Special Major Project of Forest Engineering(project number: 501035).

\section{References}

[1] Trauis Langbecker. Kinematic Analysis of Deployable Scissor Structures. International Journal of Space Structures (S0266-3511),1999,14(1):1-15.

[2] B P Nagaraj. Kinematics of Pantograph Masts. Mechanism and Machine Theory (S0094-114X), 2009, 44(4): 822-834.

[3] Sun Dongming, Dong Weimin, Li Shan. Symmetrical driving heavy-duty scissor lift platform design of mechanical design and manufacturing, 2006 6:23-24

[4] .Liu Qiaoling . Theoretical mechanics. Beijing: Science Press, 2010.

[5] song Yaojun, Liu hazel hydraulic cylinder driven scissors mechanism kinematic and dynamic analysis of lifting machinery, 2004,2: 41-43

[6] H Spackman. Mathematical Analysis of Actuator Forces in a Scissor Lift. USA: Naval Command, Control and Ocean Surveillance Center, 1994: 2-14.

[7] Suh N P. The Principle of Design.Oxford University Press,New York,1990:30-50 